\title{
Immunomagnetic detection of cancer cells in pleural effusion of generalized cancer
}

\author{
Grzegorz Kacprzak $^{1}$, Katarina Kolostova ${ }^{2}$, Jerzy Kolodziej ${ }^{1}$, \\ Ireneusz Pawlak ${ }^{1}$, Adam Rzechonek ${ }^{1}$, Vladimir Bobek ${ }^{2,3}$
}

\author{
${ }^{1}$ Department of Thoracic Surgery, Wroclaw Medical University, Wroclaw, Poland \\ ${ }^{2}$ Department of Tumor Biology, $3^{\text {rd }}$ Faculty of Medicine Charles University Prague, Czech Republic \\ ${ }^{3}$ Department of Surgery, $3^{\text {rd }}$ Faculty of Medicine Charles University and Hospital Kralovske \\ Vinohrady Prague, Czech Republic
}

\begin{abstract}
Malignant pleural effusions (MPE) are a common clinical problem in patients with neoplastic disease. Pleural fluid cytology is the simplest definitive method for obtaining a diagnosis of MPE. We describe a method that may increase the cancer cell detection rate using immunomagnetic separation in MPE. In comparison to standard MPE cytodiagnostic methods, we report a more streamlined method of isolation living cells that are able to proliferate. These captured cells can then be used for additional downstream analysis e.g. chemosensitivity testing. Several case studies of MPE diagnostics using immunomagnetic separation are presented in the following report. The immunomagnetic separation of cancer cells from MPE could be used for more accurate staging of patients with routine effusions. (Folia Histochemica et Cytobiologica 2013, Vol. 51, No. 3, 201-205)
\end{abstract}

Key words: pleural effusion, cancer cell isolation, immunomagnetic beads, cell culture

\section{Introduction}

Malignant tumors are a common cause of exudates because the serosal surfaces are a frequent site of metastases of many tumors and the site of origin for malignant mesothelioma. Malignant pleural effusions (MPE) are common clinical problems in patients with neoplastic diseases (e.g. lung, breast, and ovary carcinoma, lymphomas, mesothelioma) [1].

Usually, if MPE is reported, a documented primary tumor is on record as well. However, in a fraction of cases, effusion is the first manifestation of an unknown primary tumor. Identifying the MPE origin is essential for appropriate staging and therapy [2]. The presence of MPE correlates with a higher stage of disease and worsens prognosis.

Correspondence address: V. Bobek, $3^{\text {rd }}$ Faculty of Medicine Charles University Prague Department of Tumor Biology,

Ruska 87, 10097 Prague, Czech Republic;

tel.: +420 267102 108, fax: + 420267102 650;

e-mail: vbobek@centrum.cz
Pleural fluid cytology is the simplest definitive method for obtaining a diagnosis of MPE and it is more sensitive than blind biopsy for serosal malignancy $(71 \%$ versus $45 \%)$ [3]. However, sometimes the number of cancer cells is very low and the cells may be lost during the analytical procedures. The cancer detection rate may be increased from $2 \%$ to $38 \%$ when multiple specimens are examined by cytology [4-6]. This still leaves a substantial false-negative rate. According to statistics only $58 \%$ to $71 \%$ of MPE are diagnosed [3]. For patients with a strong clinical suspicion of pleural disease and a negative cytology result, a thoracoscopy is the procedure of diagnostic choice. Thoracoscopy could be omitted, if for the diagnosis of MPE a more sensitive method of cancer cell capture would be used. An immunomagnetic separation was proposed as a more streamlined method for routine MPE analysis [7].

MPE represents an excellent source of a wide variety of cancer cell cultures. MPEs gave rise to in vitro cultures both in adherent and/or in spheroid conditions $[1,8]$. It has been recently reported that cancer cells obtained from MPE and implanted into 
SCID mice gave rise to metastasizing tumors, which suggests indirectly the presence of tumor initiating cells in the MPE [1].

Besides the potential of MPEs being an excellent source of in vitro cultures of cancer cells, the pleural invasion score is also a very useful prognostic indicator in lung cancer. In many cases, the cancer may exfoliate itself into the pleural cavity and may progress to MPE without invading the parietal pleura. Examining pleural lavage cytology (PLC) in clinical practice is useful for detecting occult pleural dissemination before the appearance of MPE. However, the contribution of PLC to TNM staging has not been clarified yet. Evidence of PLC positive findings should be considered as supplemental information to the precise diagnosis of the pleural invasion score [9].

Therefore, one may conclude that any additional sensitivity of cancer cell isolation obtained from the pleural cavity is desirable. In this paper we report cancer cell capturing by immunomagnetic separation using paramagnetic beads with a mixture of antibodies bound on their surface. Three problems have been addressed by immmunomagnetic separation in our case report: (i) the detection of cancer cells using a mixture of antibodies, (ii) the verification of malignancy, (iii) the possibility of setting up in vitro cultures of the captured cancer cells which can later be used for downstream analysis e.g. chemosensitivity testing to personalize the cancer treatment.

\section{Material and methods}

Patients. Patient N1: A 61 year-old female with a cancer history was admitted because of right side pleural effusion of unknown origin. Two years earlier the patient underwent a left hemicolectomy and a hysterectomy for advanced colorectal adenocarcinoma. The confirmation of malignancy would mean a worsening of the patient's prognosis, but also a chance to target the chemotherapy.

Patient N2: A 57 year-old female, with no cancer record, was admitted to the hospital because of pleural effusion. The detection of cancer cells by immunomagnetic separation would help in primary tumor diagnostics. Previous patient CT-scanning did not identify any primary tumor site. Subsequently, she was diagnosed with advanced ovarian cancer.

Patient N3: A 56 year-old male was admitted because of advanced non-small cell lung cancer (NSCLC). On examination a pleural effusion was detected. Immunomagnetic separation was used for cancer cell enrichment from MPE to demonstrate the specificity of the bead-antibodies cocktail to bind surface molecules of lung cancer cells.

Cancer cell detection from malignant pleural effusion (MPE) by immunomagnetic separation. MPE $(0.5-50 \mathrm{~mL})$ was obtained by thoracic puncture. Erythrocytes were lysed by EL-buffer (Qiagen, Hamburg, Germany) and the remaining cells were pelleted by centrifugation and resuspended in $1 \mathrm{~mL}$ of Phosphate Buffer Saline (PBS, Verviers, Belgium). Immunomagnetic beads (AdnaTestBreastCancerSelect ${ }^{\mathrm{TM}}$ and/ /or AdnaTestColonCancerSelect ${ }^{\mathrm{TM}}$, Adnagen, Langenhagen, Germany) were used for cancer cell enrichment from MPE. The AdnaTest ${ }^{\mathrm{TM}}$ enables the immunomagnetic enrichment of tumor cells via bead-bound antibodies against epithelial (BerP4) and tumor-associated antigen (MUC1). A slightly modified manufacturer's protocol was applied. After $15 \mathrm{mi}-$ nutes of incubation at room temperature on the wheel rotator, the tube was placed in a Magnetic Particle Concentrator stand (DYNAL, Oslo, Norway), which caused the paramagnetic beads to attach to the wall of the tube. The attached cells were washed 3 times with $1 \mathrm{~mL}$ PBS.

In vitro cancer cell culture. The bead-captured live cells were then suspended in PBS or culture medium and analyzed immediately under microscopy (see Figure 1). The cancer cell cultures were further maintained in RPMI (Sigma-Aldrich, Schnelldorf, Germany) supplemented with $10 \%$ (v/v) fetal bovine serum (FBS (Sigma), and antibiotics (penicillin-streptomycin and amphotericin (Sigma). When a semiconfluence was reached, the cells were stained directly in the culture flasks by May-Grünwald method (see Figures 2 and 3). All microscopic observations were performed with TS100 inverted microscope (Nikon, Tokyo, Japan).

\section{Results}

The antibody-coated magnetic beads (Adnatest ${ }^{\mathrm{TM}}$ ) bind to molecules (antigens) of the cell membrane. Then, according the manufacturer's protocol, the captured cells can be isolated from peripheral blood by use of a magnetic field. The reported specificity of epithelial cell detection in peripheral blood is generally around $90 \%$ for different types of cancer if using Adnatest ${ }^{\mathrm{TM}}$.

Our results describe the use of immunomagnetic beads for rapid isolation and visualization of cancer cells from MPE in the colon cancer in the patient (N1) with primary tumor resected two years ago, in the patient with an unknown primary tumor (N2), diagnosed later with advanced ovarian cancer and in the patient with advanced NSCLC (N3). In all three cases the cancer cells were detected within 30 minutes (see Figure 1-3). Variable starting volumes of MPE were used for the immunomagnetic separation, but as reported in the methods section, the cell sediment obtained after erythrocyte lyses was resolved in all cases in the same volume of PBS $(1 \mathrm{~mL})$. The $1 \mathrm{~mL}$ of cell suspension was then incubated with $10 \mu \mathrm{L}$ of immunobeads in all presented cases.

The cancer cells, captured and visualized by cell surface-bound beads, were further propagated within 


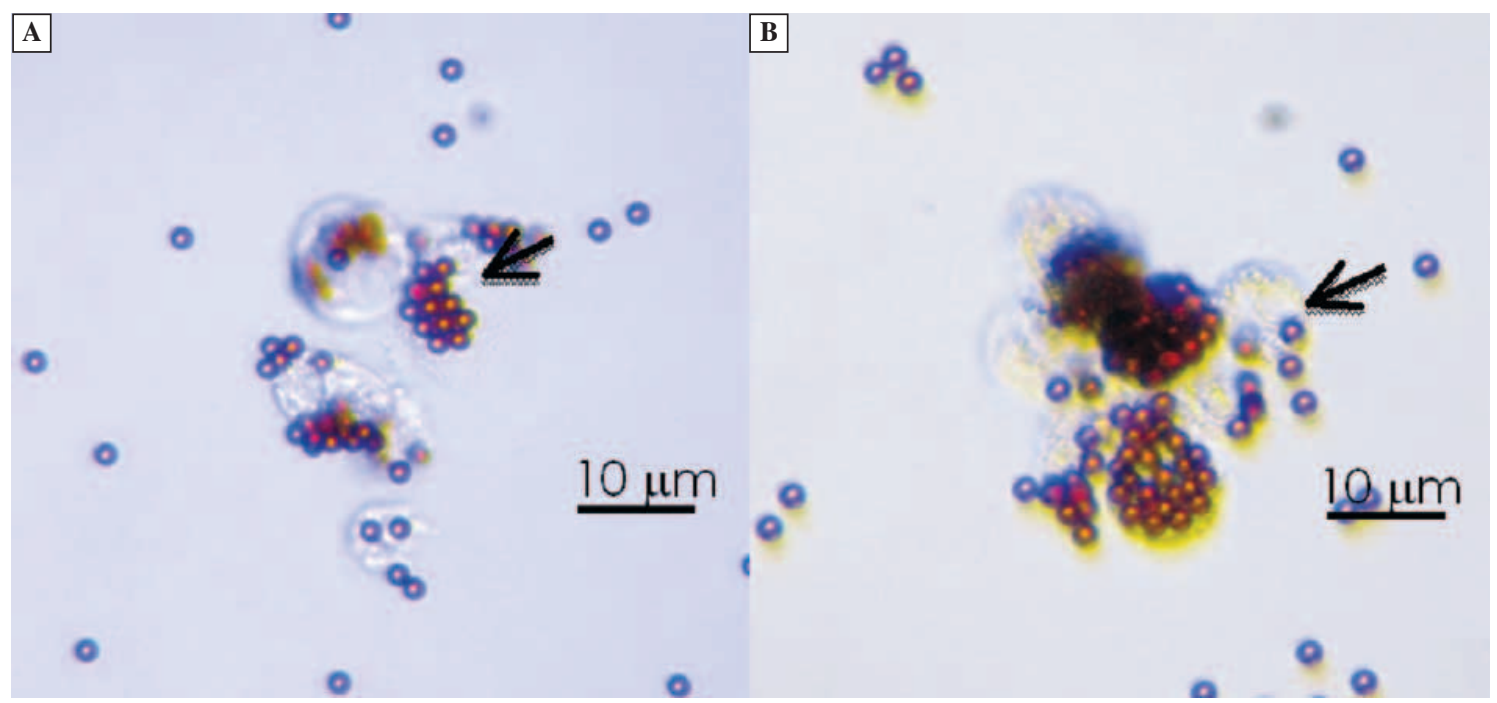

Figure 1. Cancer cells isolated from malignant pleural effusion - colorectal cancer (Patient N1). The cancer cells were captured by incubation with the immunomagnetic beads (indicated by arrows) and visualized 2 hours (A) and 4 hours (B) after the separation process (see Material and Methods). The immunomagnetic beads are distributed on the cell surface unevenly, showing a different surface molecule expression pattern. We can follow a cell adhesion process visible in Figure B

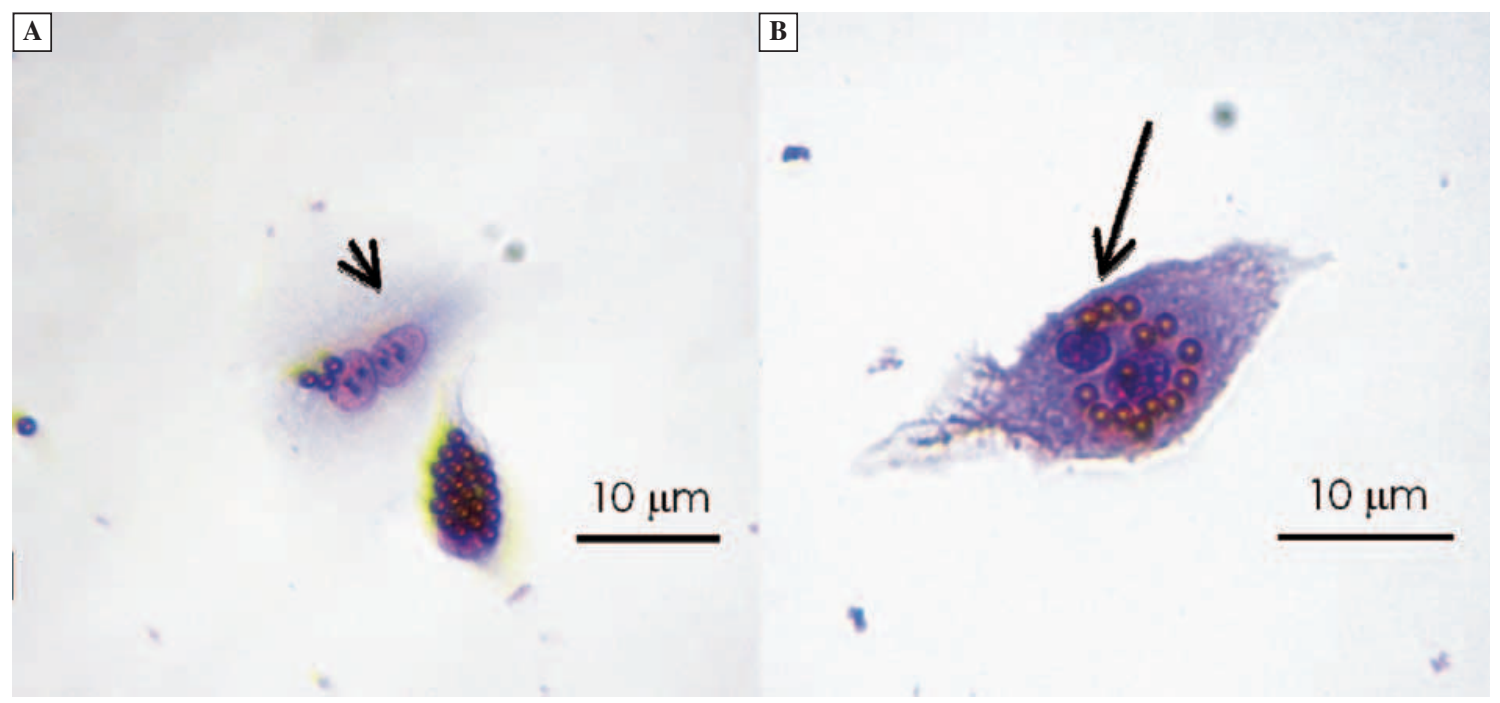

Figure 2. Cancer cells isolated from pleural effusion - ovarian cancer (Patient N2). The cancer cells are shown after in vitro incubation ( 2 weeks), stained by May-Grünwald staining. In addition to other characteristics, malignancy has been confirmed by prominent nucleoli (see arrows) in a cell with 2 nuclei (A). A detail of cancer cells with 2 nuclei (B), growing with bound beads (see arrows) indicates the proliferating potential of cancer cells, which is not influenced by the "enveloping" beads

the enriched cancer cell fraction in vitro, until they reached a semiconfluence state. The cell culture process usually took less than 14 days. The cells were further analyzed according to basic morphological features of cancer cells after May-Grünwald staining, i.e. the high nucleus/cytoplasm ratio, multiple nuclei and nucleoli, loss of contact inhibition and long term viability (see Figure 2,3). It was found that the number of cells was high enough to prepare serial slides for further immunohistochemical and mutational analysis if needed.

\section{Discussion}

The idea of identifying malignant cells in bodily fluid specimens to assess the stage of cancer patients more accurately is quite old $[1,6,7]$. The primary aim of this 


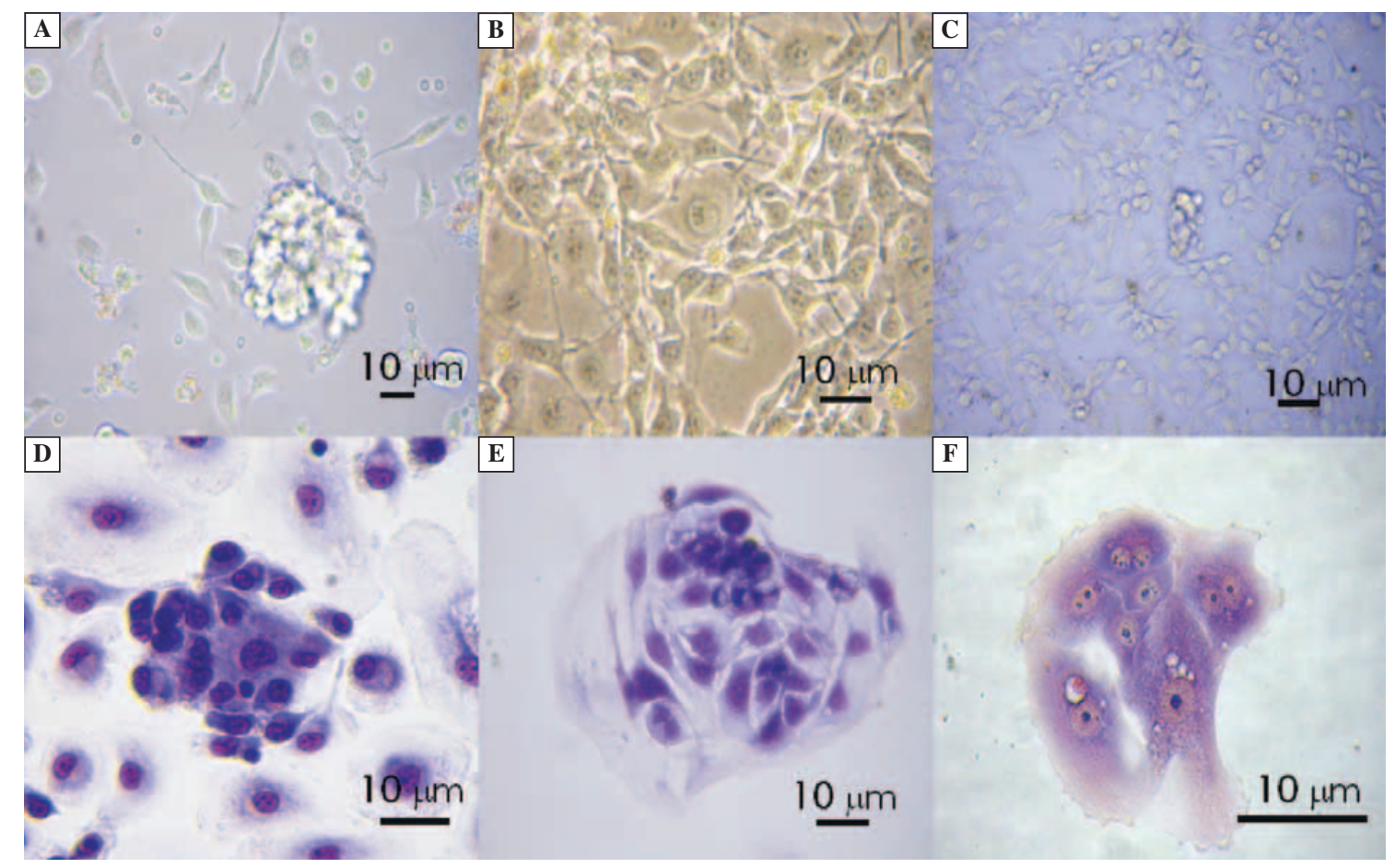

Figure 3. In vitro cancer cells cultures gained from pleural effusion. Figure A represents the MPE-derived ovarian cancer cells (Patient N2) after 2 days in vitro. Figure B, C show cell cultures derived from the same patient, but from a MPE drained one year after the first MPE-drainage. Prominent nuclei and nucleoli are visible in Figure B. Figure D, E, F show cancer cells cultured from pleural effusion of NSCLC patient, stained by May-Grünwald method (Patient N3). The staining revealed typical cancer cell morphology (high nucleus/cytoplasm ratio, multinuclear stage and rigid cytoplasm structure)

short study was to isolate a pure cancer cell fraction from the MPE of patients as quickly as possible. We focused on in vitro culturing of the captured cells for further downstream analysis. The MPE-diagnostic yield depends on factors such as the extent of the disease and the nature of the primary malignancy. Therefore, studies have shown a large variation in the diagnostic yields of MPE ranging from 62 to $90 \%$ cancer cell positivity [10,11].

As previously mentioned, there is still a substantial false negativity rate in the standard diagnostics of MPE [6]. Therefore, the enrichment technique could help in more accurate staging by the initial procedures and spare subsequent diagnostic tests or procedures [7]. In this study, we have not attempted to estimate the sensitivity of the detection of malignant cells in fluid. Analyzing only a few cases we wanted to demonstrate that the isolation process could be accurate and fast.

Magnetic beads-based separation methods have been used in the past to enrich tumor cells from blood, bone marrow, PBMC (peripheral blood mononuclear cells) fraction, ascites and effusions $[7,12,13]$. The basic principle of this method is the use of paramagnetic beads that are bound to antibodies, most frequently
EpCAM (Epithelial Cell Adhesion Molecule) and their ability to magnetic separation. EpCAMs were found to be expressed on the membrane of the majority of tumors of epithelial origin [14].

The recent reports have shown how variable the EpCAM expression can be, and, in conclusion, how cocktail of antibodies is eligible [15-18]. A combination of antibodies bound to the beads would enable the capturing of cancer cells, which do not necessarily express EpCAM as used in the Adnatest ${ }^{\mathrm{TM}}$ protocols. Cell membrane EpCAM expression may be reduced during the epithelial-mesenchymal transition (EMT) process, which is one of the ultimate conditions of the tumor dissemination process. Likewise, loss of EpCAM is coincident with vimentin overexpression (mesenchymal marker) in the MPE specimens [19].

Immunomagnetic separation, is very advantageous if there are further plans to work with the living cancer cells. It is possible to use these cells for viability testing (e.g. xCelligence System, Roche) or gene expression analysis. Other isolation protocols of disseminated tumor cells from body fluids do not make enrichment of living cancer cells possible with such specificity (e.g. gradient based separation methods). 
Other procedures, such as immunohistochemical staining with monoclonal antibodies as tumor markers, DNA and chromosome analysis have been proposed to further aid in the cytological diagnosis of MPE [20]. The relative high number of immunomagnetically enriched and cultured cancer cells can confirm the malignancy by several methods [7]. The cells are an enormous source of research material to be tested in relationship to guided oncological treatment [21]. Effusions may offer a unique in situ milieu to establish a 'pre-metastatic niche' [1]. We expect that thanks to our methodology it will be possible to test the chemosensitivity of the in vitro grown patients' MPE-enriched cells to recommend the most effective treatment. Furthermore, gene expression testing of enriched MPE-cancer cells may be used to detect multidrug resistance gene expression. Establishment of a model MPE-cancer cell culture may provide a better understanding of the role of MPE-cancer cells in metastasis with respect to the high therapeutic failure rates in patients with MPE.

In conclusion, we report a more streamlined method which makes it possible to isolate living cancer cells within 30 minutes without any impairment of cell proliferation capacity. The immunomagnetic separation of living cancer cells from MPE could be used for more accurate staging of patients with effusions.

\section{Acknowledgements}

This study was supported by the Research project $\mathrm{P}$ 27/2012 awarded by Charles University in Prague, 3rd Faculty of Medicine, Prague, Czech Republic and grant of the Czech Ministry of Health: IGA NT 14441-3/2013.

\section{References}

1. Mancini R, Giarnieri E, De Vitis Cet al. Spheres derived from lung adenocarcinoma pleural effusions: molecular characterization and tumor engraftment. PLoS One. 2011;7:21320.

2. Nasser H, Kuntzman TJ. Pleural effusion in women with a known adenocarcinoma: the role of immunostains in uncovering another hidden primary tumor. Acta Cytol. 2011;55:438-444.

3. Nance KV, Shermer RW, Askin FB. Diagnostic efficacy of pleural biopsy as compared with that of pleural fluid examination. Mod Pathol. 1991;4:320-324.

4. Motherby H, Nadjari B, Friegel P et al. Diagnostic accuracy of effusion cytology. Diagn Cytopathol. 1999;20:350-357.
5. Garcia LW, Ducatman BS, Wang HH. The value of multiple fluid specimens in the cytological diagnosis of malignancy. Mod Pathol. 1994;7:665-668.

6. Johnston WW. The malignant pleural effusion. A review of cytopathologic diagnoses of 584 specimens from 472 consecutive patients. Cancer. 1985;56:905-909.

7. Kielhorn E, Schofield K, Rimm DL. Use of magnetic enrichment for detection of carcinoma cells in fluid specimens. Cancer. 2002;94:205-211.

8. Kubo A, Koh Y, Kawaguchi T et al. Malignant pleural effusion from lung adenocarcinoma treated by gefitinib. Intern Med. 2011;50:745-748.

9. Kaneda M, Yokoi K, Ito S et al. The value of pleural lavage cytology examined during surgery for primary lung cancer. Eur J Cardiothorac Surg. 2012;41:1335-1341.

10. Weichselbaum R, Marck A, Hellman S. Pathogenesis of pleural effusion in carcinoma of the breast. Int J Radiat Oncol Biol Phys. 1977;2:963-965.

11. Fentiman IS, Rubens RD, Hayward JL. Control of pleural effusions in patients with breast cancer Cancer. 1983;52: 737-739.

12. Starr RL, Sherman ME. The value of multiple preparations in the diagnosis of malignant pleural effusions. Acta Cytol. 1991;35:533-537.

13. Antony VB, Loddenkemper R, Astoul P et al. Management of malignant pleural effusions. Eur Respir J. 2001;18:402-419.

14. Imrich S, Hachmeister M, Gires O. EpCAM and its potential role in tumor-initiating cells. Cell Adh Migr. 2012;6:30-38.

15. Rao CG, Chianese D, Doyle GV et al. Expression of epithelial cell adhesion molecule in carcinoma cells present in blood and primary and metastatic tumors. Int J Oncol. 2005;27:49-57.

16. Sieuwerts AM, Kraan J, Bolt J et al. Anti-epithelial cell adhesion molecule antibodies and the detection of circulating normal-like breast tumor cells. J Natl Cancer Inst. 2009;101:61-66.

17. Mostert B, Kraan J, Bolt-de Vries J et al. Detection of circulating tumor cells in breast cancer may improve through enrichment with anti-CD146. Breast Cancer Res Treat. 2011;1271:33-41.

18. Sterzynska K, Kempisty B, Zawierucha P et al. Analysis of the specificity and selectivity of anti-EpCAM antibodies in breast cancer cell lines. Folia Histochem Cytobiol. 2012;50:534-541.

19. Giarnieri E, De Vitis C, Noto A et al. EMT markers in lung adenocarcinoma pleural effusion spheroid cells.J Cell Physiol. 2013;228:1720-1726.

20. Rijken A, Dekker A, Taylor S et al. Diagnostic value of DNA analysis in effusions by flow cytometry and image analysis. Am J Clin Pathol. 1991;95:6-12.

21. Segal A, Sterrett GF, Frost FA et al. A diagnosis of malignant pleural mesothelioma can be made by effusion cytology: results of a 20 year audit. Pathology. 2013;45:44-48.

Submitted: 30 November, 2012 Accepted after reviews: 21 August, 2013 